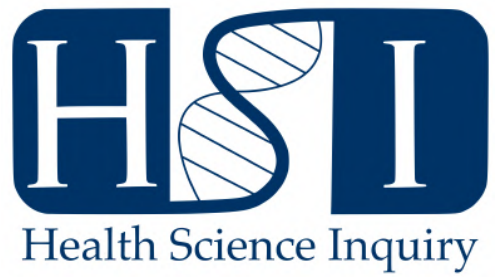

\title{
Considerations for Mitochondrial Replacement Therapy as a Novel Assisted Reproductive Technology
}

\section{Lisa Oliviero ${ }^{1}$ \\ ${ }^{1}$ Division of Experimental Medicine, McGill University}

Mitochondria are essential organelles of nucleated cells [1]. While most of the DNA contained in a cell is located within the nucleus, mitochondria, located in the cytoplasm, contain a small fraction of an organism's overall DNA [1]. The fraction of DNA located in the mitochondria is called mitochondrial DNA (mtDNA) [1]. This particular type of DNA, which is distinct from nuclear DNA, counts for less than 0.1 percent of the total amount of genetic material within a human cell [1]. Despite its minor presence, defects in the mtDNA can cause significantly harmful pathologies, namely mitochondrial diseases, as the mitochondria are in charge of providing energy to the entire cell [1]. Two commonly recognized mitochondrial diseases include myoneurogastrointestinal encephalopathy syndrome (MNGIE), which features myopathy, neuropathy, chronic malnutrition, and sensorineural hearing loss, and Leigh syndrome, which is proven to have a very grim prognosis, with death occurring within the first years of life [2].

Considering mitochondria are maternally inherited, a recent scientific innovation has emerged to prevent the transmission of defective maternal mtDNA to offspring. This recent development is referred to as mitochondrial replacement therapy (MRT). This form of assisted reproductive technology encourages an option for women carrying mutated mtDNA to have genetically related children without passing down their mtDNA [1]. MRT functions by primarily retrieving healthy mitochondria from an egg donor, and can proceed with either of the following techniques (Figure 1): In the first technique, pronuclear transfer, the transfer of mtDNA follows the fertilization of the egg that has occurred in vitro [1]. The two eggs (one from the intending mother and one from the donor) are fertilized with the intending father's or another donor's sperm [1]. Shortly after fertilization takes place, the pronucleus of the embryo containing the mother's mitochondria is transferred to a previously enucleated embryo containing healthy donor mitochondria [1]. In the second technique, maternal spindle transfer, the healthy nucleus of an egg with the affected mitochondria is removed and then transferred to the egg of the donor containing healthy mitochondria, which has been previously deprived of its nucleus [1].

While there is an evidentiary basis for the technical feasibility of MRT documented for both human embyros and

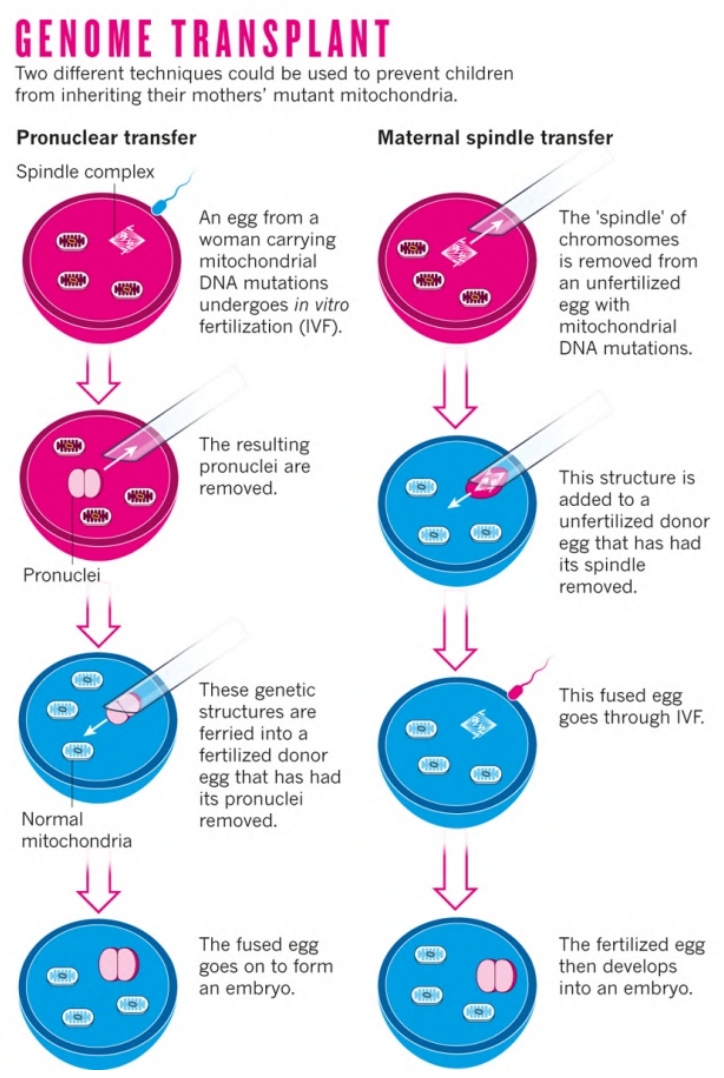

Figure 1: MRT techniques for prevention of mutated mtDNA transmission. Adapted from [3]

embryonic stem cells, questions have remained regarding whether a "mismatch" between mtDNA and nuclear DNA may cause mitochondrial dysfunction in children [4]. An important research milestone was achieved when studies demonstrated the birth of healthy monkey infants following MRT. In a postnatal analysis, their overall health monitored from birth to 3 years was comparable to the age- 
matched controls [4]. These results evidently pave a promising path for further confirmatory studies to be completed, and when appropriate, application to human populations.

Although this technology could offer significant therapeutic potential, MRT remains illegal in Canada and across other jurisdictions [5]. Much legal and ethical controversies of MRT hover around a concern for inappropriately modifying the genetic material of germline cells that can be passed on to offspring, and their subsequent offspring. In Canada, the Assisted Human Reproduction Act (AHRA) states in section 1f that "no person shall knowingly alter the genome of a cell of a human being or in vitro embryo such that the alteration is capable of being transmitted to descendants" [5]. Despite legal support in the United Kingdom (UK) for early phase clinical trials based on studies in animals and human oocytes, the criminal ban in Canada on human germline alterations is considered to apply to MRT and may foreclose any possibility of pursuing similar MRT trials within the jurisdiction [6]. However, MRT restriction raises ethical concerns as there is a strong therapeutic potential associated with its application.

The UK and the United States (US) are the only countries moving forward with this technology [7]. In the UK, after years of discussion guided by the Human Fertilization and Embryology Authority, Parliament approved the licensed clinical use of both MRT techniques in October of 2015 [7]. With this progress, clinical application is deemed to be imminent [7]. In the US, the Food and Drug Administration (FDA) is delaying approval until more preclinical data is collected. Further, they have specified that MRT would be reserved only to create male embryos to preclude the transmission of donor mtDNA to future generations, thereby bypassing the germline modification.

Evidently, there are ongoing efforts in certain jurisdictions to surface this technology from the preclinical realm to the clinical domain. Although Canada is behind on this progress, it is important to ponder how we would proceed in providing MRT if legalized, and which individuals would have accessibility. This would be a significant advancement in allowing women with mitochondrial diseases to have genetically related, without passing down their affected mtDNA. Nonetheless, further research ought to be carried out in order ensure this technology yields utmost therapeutic benefit, while minimizing any harms to mothers and their children.

\section{REFERENCES}

[1] Castro RJ. Mitochondrial replacement therapy: the UK and US regulatory landscapes. Journal of Law and the Biosciences. 2016;3(3):726.

[2] Gropman AL. Diagnosis and treatment of childhood mitochondrial diseases. Current neurology and neuroscience reports. 2001;1(2):185-194.

[3] Callaway E. Reproductive medicine: The power of three. Nature News. 2014;509(7501):414.

[4] Tachibana M, Amato P, Sparman M, Woodward J, Sanchis DM, Ma H, et al. Towards germline gene therapy of inherited mitochondrial diseases. Nature. 2013;493(7434):627.

[5] Branch, LS. Consolidated federal laws of Canada, Assisted Human Reproduction Act; 2012. Available from: https://laws-lois.justice.gc.ca/eng/acts/ a-13.4/page-1.html\#h-2.

[6] Knoppers BM, Leader A, Hume S, Shoubridge EA, Isasi R, Noohi F, et al. Mitochondrial replacement therapy: The road to the clinic in Canada. Journal of Obstetrics and Gynaecology Canada. 2017;39(10):916-918.

[7] Klopstock T, Klopstock B, Prokisch H. Mitochondrial replacement approaches: challenges for clinical implementation. Genome medicine. 2016;8(1):126.

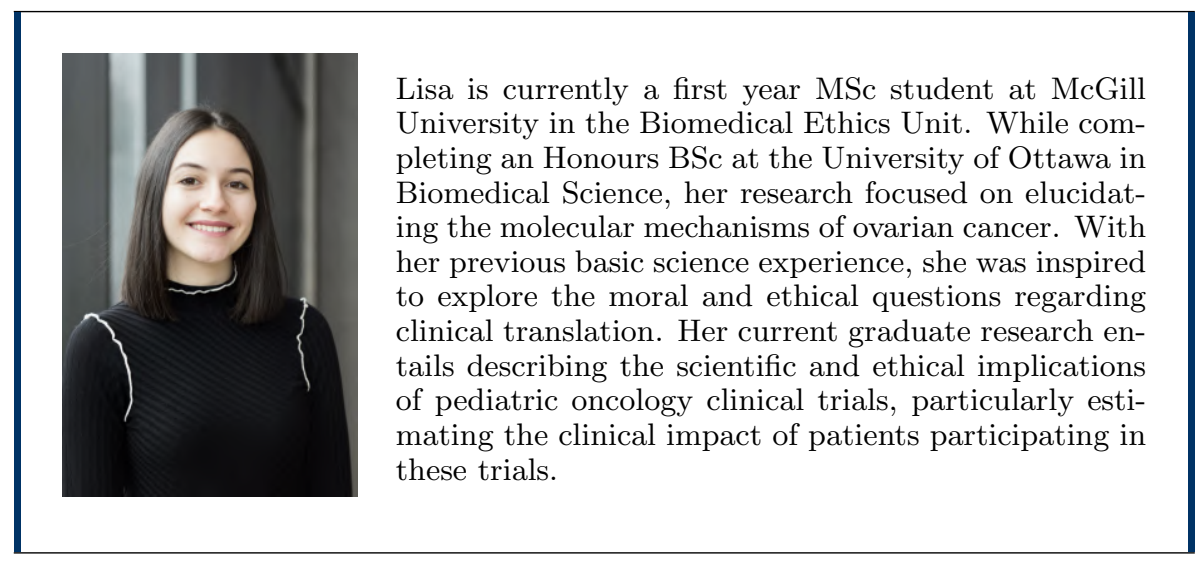

